\title{
Considerações teóricas sobre a relação entre respiração oral e disfonia
}

\section{Theoretical considerations on the relationship between mouth breathing and dysphonia}

\author{
Juliana Gomes Tavares ${ }^{1}$, Erika Henriques de Araújo Alves da Silva²
}

\begin{abstract}
RESUMO
Sabe-se que a respiração oral pode ocasionar diversas alterações ósseas, posturais, musculares e funcionais, como também, alterações na qualidade e/ou comportamento vocal. Estas alterações podem comprometer a comunicação oral, na qual a voz não consegue desempenhar seu papel básico de transmissão da mensagem verbal e emocional do indivíduo. O indivíduo com respiração oral pode apresentar ressonância nasalizada e a voz pode apresentar alterações no traço de sonoridade, hiper ou hiponasal, ou rouca. A partir de pesquisa nas bases de dados LILACS, SciELO, livros, revistas especializadas sobre o tema, buscamos fazer uma revisão na literatura sobre a relação entre respiração oral e disfonia, analisando a influência das alterações causadas pela respiração oral na qualidade e/ ou comportamento vocal. No entanto, foi possível observar que a respiração oral promove uma série de alterações estruturais significativas que refletem nas funções estomatognáticas, interferindo também na fonação. Contudo, poucos autores relatam a possível correlação entre esses dois parâmetros (respiração oral e disfonia).
\end{abstract}

Descritores: Voz; Respiração bucal/complicações; Disfonia; Qualidade da voz

\section{INTRODUÇÃO}

Voz e Motricidade Oral são áreas de especialidade que ocupam lugar de destaque na Fonoaudiologia. Ambas estudam o aparelho fonador em suas dimensões constitucionais, neurológicas e funcionais ${ }^{(1)}$.

As funções estomatognáticas são comportamentos realizados a partir das estruturas que compõem o sistema estomatognático, ou seja, os ossos fixos da cabeça, a mandíbula, o hióide e o esterno; os músculos da mastigação, da deglutição e faciais; a articulação temporomandibular (ATM); os dentes e tecidos anexos; o sistema vascular e também que dependem do sistema nervoso central e periférico ${ }^{(2)}$.

São consideradas funções estomatognáticas a mastigação, a sucção, a deglutição, a fala e a respiração. Todas as funções atuam de acordo com suas propriedades funcionais e seus sistemas específicos de controle; porém, é necessária

Trabalho realizado no Curso de Fonoaudiologia da Universidade Estadual de Ciências da Saúde de Alagoas - UNCISAL - Maceió (AL), Brasil.

(1) Especializanda em Motricidade Orofacial pela Faculdade Integrada do Recife - FIR - Recife (PE), Brasil.

(2) Pós-graduanda em Distúrbios da Comunicação Humana pela Universidade Federal de São Paulo - UNIFESP - São Paulo (SP), Brasil; Professora do Curso de Fonoaudiologia da Universidade Estadual de Ciências da Saúde de Alagoas - UNCISAL - Maceió (AL), Brasil.

Endereço para correspondência: Juliana Gomes Tavares. R. "P", 140, Conjunto Cidade Universitária, Tabuleiro dos Martins, Maceió - AL, CEP 57072-033. E-mail: juli_fono22@yahoo.com.br

Recebido em: 30/11/2007; Aceito em: 1/7/2008 uma inter-relação harmônica entre eles, que finaliza em um equilíbrio do sistema ${ }^{(3)}$.

A respiração é o mecanismo fisiológico que permite a ocorrência das trocas entre o organismo e o ambiente. Quando há interferência ou desvio no padrão de respiração nasal, por manifestação de determinados fatores etiológicos, ocorre a suplência oral ou respiração mista ${ }^{(4)}$.

Os fatores etiológicos da respiração oral podem ser alterações alérgicas como: rinite ou sinusite; alterações anatômicas na cavidade nasal, como: desvio do septo nasal, hipertrofia de cornetos, hipertrofia de adenóides, hipertrofia das amígdalas palatinas $^{(5-8)}$.

A voz falada se utiliza de estruturas do aparelho respiratório e do digestivo, na medida em que ocorrem alterações constantes na movimentação dos lábios, língua, véu palatino e da própria laringe ${ }^{(9)}$.

O sistema respiratório funciona como o ativador da voz, em que os volumes e a pressão do fluxo de ar expirado, passam pelas pregas vocais que se aproximam e põe-se em vibração, o que produz a voz. Portanto, qualquer comprometimento da função aérea pode provocar um efeito direto sobre a fala e sobre a voz (intensidade, altura, qualidade) ${ }^{(10)}$.

Em respiradores orais as funções de fala, mastigação, deglutição e voz podem estar alteradas. Todavia, a voz, muitas vezes, passa despercebida no momento da avaliação( ${ }^{(5)}$. A voz apresenta um desequilíbrio ressonantal e de coordenação pneumo-fônica-articulatória ${ }^{(11)}$. Nesses indivíduos, a voz pode, ainda, encontrar-se com uma alteração no traço de sonoridade, hiper ou hiponasal, ou rouca ${ }^{(3,6)}$. 
A disfonia pode ser qualquer dificuldade na emissão vocal que impeça a produção natural da voz ${ }^{(12)}$. Os distúrbios da voz resultam de alterações nas estruturas ou no funcionamento do trato vocal: na respiração, na vocalização ou na ressonân$\mathrm{cia}^{(10)}$. Podem-se citar como fatores etiológicos da disfonia as alterações congênitas da laringe, infecções de vias aéreas, fatores alérgicos, obstrução nasal, abuso vocal, hábitos vocais inadequados, dentre outros ${ }^{(11,13-14)}$.

O objetivo do presente estudo é fazer uma revisão na literatura sobre a relação entre respiração oral e disfonia, analisando a influência das alterações causadas pela respiração oral na qualidade e comportamento vocal. Para tal, foi realizada uma pesquisa bibliográfica, nas bases de dados LILACS, SciELO, livros, revistas especializadas e monografias sobre o tema.

\section{REVISÃO DA LITERATURA}

\section{Respiração}

O sistema respiratório é um conjunto formado por órgãos tubulares e alveolares situado na cabeça, pescoço e cavidade torácica, responsável pela respiração, que são trocas gasosas efetuadas entre o organismo e o meio ambiente ${ }^{(3)}$.

A cavidade nasal tem como função filtrar, umedecer e aquecer o ar inspirado. Além de auxiliar na produção da fala. É na cavidade nasal que se encontra a primeira barreira imunológica contra agentes agressores ${ }^{(15)}$. A via respiratória é recoberta por tecido linfóide, formando o anel de Waldeyer, que é constituído, principalmente, pelas tonsilas linguais, palatinas, faríngea e tubárias, além dos tecidos linfóides ${ }^{(16)}$.

A respiração nasal propicia um desenvolvimento e crescimento do complexo craniofacial normal, favorecendo a harmonia estrutural e funcional do indivíduo ${ }^{(5)}$. O indivíduo respirador nasal mantém seus lábios ocluídos e a mandíbula encontra-se na posição de repouso. A língua fica contida na cavidade bucal propriamente dita e, pelo contato com o palato, realiza forças de expansão na maxila, que se equilibra com as forças restritivas do músculo bucinador. A coluna cervical apresenta uma curvatura fisiológica e a cabeça se mantém ereta ${ }^{(2)}$.

A respiração nasal adequada é importante para o bom funcionamento da laringe, faringe, deglutição e sensação de bem estar ${ }^{(17)}$.

A obstrução nasal conduz à respiração oral, resultando em posição alterada da língua e lábios entreabertos ${ }^{(18)}$. Devido a esta obstrução, que pode ser causada por anormalidades estruturais, patologias nasossinusais ou hipertrofia do anel linfático de Waldeyer ${ }^{(7)}$, o indivíduo adota o padrão respiratório oral. O padrão respiratório inadequado induz às adaptações funcionais, promovendo desequilíbrio muscular da face, modificações posturais, como lábios entreabertos, extensão posterior da cabeça e um posicionamento mais inferior da mandíbula e da língua ${ }^{(19)}$.

$\mathrm{O}$ aumento de volume das tonsilas e a obstrução de vias aéreas também estão associados a alterações ortognáticas, a mastigação, a deglutição e a deformidades da arcada dentária ${ }^{(20)}$.

O complexo craniofacial busca uma posição mais favorável para realizar as funções e, portanto, se adapta, conforme suas possibilidades, com a finalidade de buscar o que é mais fácil para realizar a respiração ${ }^{(19)}$.
A respiração oral pode ter vários agentes etiológicos como: rinite alérgica e não-alérgica; sinusite; desvio de septo; alergias; hipertrofia de cornetos, adenóides e amígdalas palatina $^{(3,6,15,21-22)}$.

Os fatores alérgicos das vias aéreas superiores e a inalação de ar mal condicionado, imposta pela respiração oral permanente, predispõem ao ressecamento da mucosa das vias aéreas e à inalação de micropartículas não filtradas pelas fossas nasais, facilitando as infecções recorrentes do trato respiratório superior ${ }^{(23)}$. Dependendo do tempo de respiração oral, o ressecamento na cavidade oral pode ser maior ou menor ${ }^{(6)}$.

O fato do fluxo aéreo não ser conduzido pelo nariz deve ser considerado condição patológica, e não uma alternativa fisiológica ${ }^{(3,15,24)}$.

Por apresentar vários sintomas característicos, entre eles: alterações do sistema sensório-motor e das funções orais do sistema estomatognático e, por apresentar vários fatores etiológicos, alguns autores denominaram tal alteração de Síndrome do Respirador Oral ${ }^{(8,25)}$. Estas alterações podem levar, também, a um comprometimento na $\mathrm{voz}^{(25)}$.

A alteração na função respiratória, geralmente, pode levar à ressonância nasalizada e a voz pode apresentar alterações no traço de sonoridade, hiper ou hiponasal, ou rouca ${ }^{(3,6)}$.

\section{Voz}

"A voz é o meio ao qual o ser humano extravasa seus sentimentos, sensações e impressões; é o maior canal de comunicação interpessoal e reflete o que somos e como pensamos"(11).

Para a produção da fala contribuem três processos: o mecanismo de fole utilizando o ar oriundo dos pulmões, a geração do som na glote através da vibração das pregas vocais, a ressonância e a articulação deste som, as quais ocorrem no segmento supraglóti$\mathrm{co}^{(26)}$. No entanto, a fonação não é apenas o resultado da atividade dentro da laringe. A atividade muscular do corpo inteiro é também responsável pela função apropriada da mesma ${ }^{(27)}$.

A voz depende fundamentalmente da atividade muscular de todos os músculos que servem à produção vocal, além da integridade de todos os tecidos do aparelho fonador ${ }^{(12)}$.

A laringe é um órgão evolvido na respiração e na fonação, situado logo acima da traquéia. Constitui-se por: osso hióide, membrana tireóidea, epiglote, pregas ventriculares, pregas ariepiglóticas, ventrículo de Morgagni, cartilagens e pregas vocais ${ }^{(28)}$.

Para produção vocal a laringe é o órgão essencial, juntamente com a musculatura das costas e abdômen, caixa torácica, pulmões, faringe, cavidade oral e nasal. Todas as estruturas são importantes na produção vocal e podem ser responsáveis por disfunções vocais ${ }^{(29)}$.

A voz, originada pelo fluxo de ar vibrando as pregas vocais, é amplificada nas cavidades da via aérea superior do pescoço e cabeça. Esta amplificação denomina-se ressonância ${ }^{(10)}$.

O trato vocal, em comprimento, se estende desde a glote até os lábios. O som glótico inicial é modificado pela ressonância e posteriormente pelo movimento dos articuladores ${ }^{(26)}$.

Durante a fonação, o modo respiratório costuma ser misto preferencialmente, devido à necessidade da rápida tomada de $\operatorname{ar}^{(9)}$. 
Alterações no nível respiratório, glótico e ressonantal são frequentemente encontradas na disfonia funcional, que pode ser provocada por uso incorreto, gerado por um simples desvio no processo básico da produção natural da $\operatorname{voz}^{(12)}$. Os distúrbios da voz resultam de estruturas ou funcionamento defeituosos no trato vocal: na respiração, na vocalização ou na ressonância ${ }^{(10)}$.

A disfonia pode ser definida como sendo qualquer dificuldade na emissão vocal que impeça a produção natural da $\mathrm{voz}^{(12)}$. Podem-se citar como fatores etiológicos da disfonia as alterações congênitas da laringe, infecções de vias aéreas, fatores alérgicos, obstrução nasal, abuso vocal, hábitos vocais inadequados, dentre outros ${ }^{(11,13-14)}$.

A disfonia pode manifestar-se por meio de uma série de alterações, como: esforço à emissão, dificuldade em manter a voz, cansaço ao falar, variações na freqüência fundamental habitual, rouquidão, falta de volume e projeção, perda da eficiência vocal e pouca resistência ao falar, entre outras ${ }^{(30)}$.

As alterações mais encontradas na disfonia são: nível respiratório (inspiração insuficiente para fonação, ou início fonatório após a expiração); nível glótico (uso hipertônico da compressão glótica e o uso hipotônico menos comum) e nível ressonantal (não-aproveitamento das caixas de ressonân(cia) ${ }^{(12)}$. Na disfonia, podem se encontrar, também, distorções do sinal laríngeo e desequilíbrio no foco ressonantal, que se relacionam com a região do trato vocal a qual predomina a amplificação da voz ${ }^{(31)}$.

\section{Relação entre respiração oral e disfonia}

É importante compreender a ação interdisciplinar na atuação com o respirador oral, uma vez que estes podem apresentar assimetrias faciais, problemas posturais, alterações oclusais e mau funcionamento dos órgãos fonoarticulatórios, entre outros ${ }^{(3)}$.

A relação entre distúrbios vocais e alterações das vias aéreas superiores engloba, especialmente, modificações no sistema de ressonância e de projeção vocal, gerando esforço laríngeo como mecanismo compensatório ${ }^{(1)}$.

A postura, a tonicidade e a mobilidade dos órgãos fonoarticulatórios influem diretamente na articulação e interferem na projeção vocal, nos ajustes: laríngeo e faríngeo e, portanto, têm relação com a qualidade vocal e o sistema de ressonância ${ }^{(32)}$.

Várias são as características do respirador oral; dentre elas, pode-se descrever: aumento vertical do terço inferior da face, arco maxilar estreito, posição do osso hióide mais baixa, ressecamento da mucosa oral, alteração das pregas vocais, hipotonia da musculatura elevadora da mandíbula, alteração do tamanho e posição de língua, diminuição da pressão intra-oral, alteração da posição de cabeça e pescoço, tensão da musculatura constritora da faringe, musculatura abdominal flácida e distendida, voz com hiper ou hiponasalidade ou rouca, dentre outras ${ }^{(6,8,19,33)}$.

Em uma pesquisa ${ }^{(34)}$ que levantou as relações existentes entre os distúrbios vocais e os distúrbios musculares orais, foi observado que nos casos em que a respiração nasal não esteja estabelecida, notam-se alterações musculares orais e/ ou alterações vocais, sendo estas últimas, alterações: orgânica ou funcional.
O distúrbio vocal pode decorrer de um conjunto de fatores causais, tendo cada um destes um papel de contribuição para a disfonia. É relevante ressaltar que a rinite alérgica é um importante comprometedor das condições do trato vocal, pois envolve nariz, ouvidos e garganta, o que, no mínimo, comprometerá a boa projeção da voz ${ }^{(32)}$.

Em estudo foi realizado para verificar a incidência de sinais e sintomas vocais em pacientes adultos com diagnóstico de rinite alérgica, comparando-os com grupo-controle ${ }^{(35)}$. Foi aplicado um questionário com 30 questões fechadas sobre os hábitos, sintomas e sinais vocais, para o grupo de pacientes com diagnóstico de rinite e para o grupo-controle. Os autores observaram que os sintomas vocais mais freqüentes nos pacientes com rinite alérgica foram: rouquidão, pigarro, tosse e secura na boca e/ou garganta, e os sinais vocais mais freqüentes foram: voz hiponasal, mais fraca e mais grave. Na avaliação subjetiva das vozes dos pacientes, os autores observaram um aumento na incidência de voz disfônica associada a rinites mais graves.

Os respiradores orais apresentam uma série de modificações nas estruturas envolvidas no processo respiratório, alterando o crescimento e postura normal da cabeça ${ }^{(5)}$. Há uma rotação posterior do crânio e a cabeça adota uma postura estendida para favorecer a entrada de ar pela cavidade oral. $\mathrm{O}$ respirador oral assume uma postura habitual com rotação posterior do crânio para favorecer a entrada de ar, conseqüentemente, ocorre aumento da cifose torácica com aproximação dos ombros (rotação anterior) e fechamento do tórax, o que desencadeia alteração do ritmo respiratório. Apresenta, também, cabeça anteriorizada em relação ao corpo; ântero-versão do quadril e hipertextensão dos joelhos e; afastamento dos pés, visando o equilíbrio ${ }^{(6,33)}$.

A adequada postura corporal tem sido apontada como base de uma boa produção da voz e da fala, influindo até mesmo no suporte respiratório. As alterações posturais de cabeça limitam os movimentos de mandíbula e da própria cabeça, interferindo na produção vocal ${ }^{(32)}$.

A qualidade vocal é um conjunto de características que identificam individualmente a voz. O mecanismo de produção dos sons da fala requer fonte de energia, elementos vibrantes e sistema de válvulas e filtros ${ }^{(1)}$.

Quando o indivíduo adota o tipo de respiração clavicular, ocorre uma tensão de toda a região cervical ${ }^{(5)}$. O músculo esternocleidomastoídeo constantemente encontra-se tenso em disfônicos, por manter a caixa torácica elevada ${ }^{(12)}$. Sendo assim, ocorre tensão no esternocleidomastoídeo, gerando dores no pescoço ${ }^{(32)}$. Com a tensão desencadeada nesta região ocorre prejuízo na qualidade e comportamento vocal.

Entre as crianças disfônicas, o tipo respiratório superior (ou clavicular) com expansão da caixa torácica e elevação dos ombros, costuma ocorrer inadequadamente durante a fonação ${ }^{(13)}$.

Indivíduos com disfonia de base funcional muitas vezes apresentam alterações posturais, quando se percebe o tronco caído para frente, que causa compressão da região diafragmática, impedimento da expansão da musculatura respiratória, dificultando o apoio respiratório ${ }^{(32)}$. Estas alterações posturais não prejudicam apenas o posicionamento da laringe no pes- 
coço, mas também o mecanismo respiratório e processo vocal como um todo ${ }^{(31)}$.

A dinâmica respiratória da criança que apresenta uma hipercinesia vocal costuma se caracterizar por inspirações superficiais e ruidosas e pelo controle insuficiente da expiração ${ }^{(13)}$.

A musculatura extrínseca da laringe, também, exerce papel fundamental no equilíbrio do tônus necessário para desempenho das funções estomatognáticas. A hipertonicidade desses músculos pode ser um fator importante nos casos de disfonia por tensão muscular ${ }^{(1)}$. Além disso, interfere nos sistemas respiratórios, digestivo, fonatório e articulatório, permitindo concluir que qualquer movimento das estruturas interligadas com essa musculatura afetará, conseqüentemente, a forma das pregas vocais $^{(36)}$.

A extensão da cabeça habitualmente encontrada no respirador oral implica mudança de postura da mandíbula com o seu abaixamento, uma vez que a postura mandibular está relacionada com a unidade cabeça/pescoço ${ }^{(5)}$. Quando a cabeça está hiperestendida, a protrusão de língua tende a diminuir a freqüência fundamental da $\mathrm{voz}^{(1)}$.

Foi observado que as alterações miofuncionais mais comuns, nos respiradores orais, foram: a posição dos lábios entreabertas, a da língua em soalho bucal, a flacidez dos lábios, da língua e dos músculos mastigatórios e a interposição da língua durante a deglutição e a fonação ${ }^{(16)}$.

A língua e as estruturas da laringe estão intimamente conectadas ao osso hióide e movimentam-se constantemente durante a fala. Uma língua baixa, hipercinética apoiada firmemente contra os arcos dentais com o rebordo lingual marcado e sulcado pelos dentes, pode ser um sinal secundário de disfonia por Síndrome de Tensão Muscular ${ }^{(12)}$.

A posição da língua na cavidade oral influencia na ressonância da voz. O corpo da língua em posição anterior e elevada produz uma voz infantilizada, já a língua numa posição hiperfuncional, isto é, posteriorizada, produz uma ressonância posterior ${ }^{(10)}$.

As cavidades de ressonância começam dentro da própria laringe, estendem-se para a faringe, a cavidade oral e a cavidade nasal $^{(10)}$. Estas estruturas, além de serem partes intrínsecas do mecanismo da respiração, também são essenciais para articulação dos sons e para ressonância ${ }^{(1)}$. Ao nível ressonantal, o não aproveitamento das caixas de ressonância como também o direcionamento do fluxo aéreo para uma caixa de ressonância específica são fatores que estorvam a estética vocal ${ }^{(12)}$.

Faringe, língua, palato, cavidade oral, nariz e outras estruturas compõem o trato vocal supraglótico. Estas estruturas juntas agem como cavidades de ressonância e são em grande parte responsáveis pela qualidade vocal. O trato vocal infraglótico funciona como fonte de força para a vocalização, podendo-se observar que alterações na função muscular ou estrutura resultam em esforço compensatório na musculatura da laringe, podendo resultar fadiga vocal, formação de nódulos e dor ${ }^{(29)}$.

São características faciais do respirador oral: aumento vertical do terço inferior da face, rotação do ângulo da mandíbula, má oclusão dentária, posição do osso hióide mais baixa, palato em ogiva, lábio inferior com eversão, lábio superior curto, hipotonia da língua, alterações da postura da língua em repouso, narinas estreitas, flacidez da musculatura orofacial ${ }^{(15,21,37-38)}$.
Os indivíduos com características dolicofaciais tendem a apresentar qualidade vocal abafada, escura e associada à ressonância posterior ${ }^{(39)}$.

A produção vocal tem importância primordial no relacionamento entre a estrutura esqueletal, a oclusão dentária e o tecido muscular que recobre a face em toda sua forma, dimensão e tensão ${ }^{(39)}$.

\section{DISCUSSÃO}

O interesse por este trabalho surgiu a partir da observação do número de pacientes que apresentam alterações na respiração, qualidade e/ou comportamento vocal, respiração oral e distúrbios vocais, observando assim a relação existente entre essas duas vertentes. Também, foi observada a inter-relação anatômica e fisiológica das estruturas que compõem as funções de respiração e fonação.

Considerando a literatura pesquisada foi observado que, na presença de alterações respiratórias, outras dificuldades são encontradas. Porém, poucos são os estudos que correlacionam respiração oral e alterações na qualidade e/ou comportamento vocal.

A musculatura envolvida na fonação necessita trabalhar de forma sinérgica, com o objetivo de produzir uma voz equilibrada. No entanto, pode-se notar que quando o respirador oral apresenta alteração na postura corporal, a fim de facilitar a entrada de ar pela cavidade oral, ele acaba por sobrecarregar a musculatura supra e infra-hióidea, modifica a posição da língua, entre outras, o que pode levar o indivíduo desenvolver as alterações vocais e ressonantais.

No respirador oral, a modificação postural torna-se acentuada na medida em que a postura da cabeça é estendida, o que dificulta a sua precisão articulatória. Além da postura, modificações das estruturas do sistema estomatognático, também, influenciam na precisão articulatória, bem como, na qualidade e/ou comportamento vocal.

A partir do levantamento bibliográfico, observou-se que a forma do esqueleto craniofacial e as características musculares e funcionais podem ter influência na qualidade vocal. No respirador oral, indivíduos de face longa, a qualidade vocal tende a ser abafada, escura e associada à ressonância posterior, sendo que estas diferenças parecem ter origem na forma e configuração do trato vocal ${ }^{(39)}$.

O fato dos músculos que compõem o aparelho fonador serem os mesmos do sistema estomatognático, reforça a hipótese de que alterações posturais e/ou de tonicidade nestes músculos interferem na realização das funções da articulação (fala) e da fonação, dado significante e confirmado pela literatura ${ }^{(34)}$.

Nas disfonias funcionais infantis, o sistema de ressonância freqüentemente se apresenta desequilibrado, pois não existe uma distribuição adequada de energia sonora entre as diferentes cavidades de ressonância ${ }^{(13)}$.

É importante salientar que as alergias respiratórias e infecções nas vias aéreas superiores fazem com que o uso da voz se dê em condições inadequadas, ou seja, na presença de mucosas ressecadas, edemas e irritação do trato vocal.

As queixas de obstrução nasal associadas aos nódulos vocais têm sido destacadas por alguns autores entre os fatores pre- 
disponentes, no desenvolvimento dos nódulos vocais ${ }^{(17,23)}$.

Pesquisadores avaliaram as relações entre os exames: otorrinolaringológico, cinefluoroscopia e análise perceptual auditiva, efetuados em crianças com indicação de adenoidectomia. Concluíram que o grau de hiponasalidade na qualidade vocal está relacionado diretamente ao grau de obstrução nasal ${ }^{(40)}$.

Em estudo realizado para avaliar a incidência de alterações obstrutivas nasais e faríngeas em 208 pacientes com nódulos vocais, foi observado que 113 casos $(54,3 \%)$ apresentaram alteração obstrutiva nasal, chamando atenção para a importância da respiração nasal no tratamento fonoaudiológico eficaz ${ }^{(17)}$. Em outros estudos realizados, foi observado que alterações de vias aéreas superiores como: rinite alérgica, hipertrofia do anel de Waldeyer, dentre outras, interferem na qualidade vocal $^{(13,40)}$. Com isso, pode-se observar que as alterações do estado geral, alergia e infecções otorrinolaringológicas podem favorecer ou acentuar o mau uso vocal. No caso das infecções viróticas das vias aéreas superiores agravam, em diferentes graus, a disfonia ${ }^{(17)}$.

Na literatura pesquisada, observamos que a obstrução nasal é fator predisponente ao surgimento da disfonia; porém, o mau uso e/ou abuso vocal continuam sendo os principais fatores para o surgimento da disfonia ${ }^{(13,17)}$.

Sabe-se que a respiração oral pode ocasionar diversas alterações ósseas, posturais, musculares e funcionais, como por exemplo, a disfonia. Esta pode comprometer a comunicação oral, na qual a voz não consegue cumprir seu papel básico de transmissão da mensagem verbal e emocional do indivíduo ${ }^{(30)}$.

Para uma boa produção vocal é necessário que haja uma postura corporal adequada, boa mobilidade da caixa torácica, integridade dos sistemas muscular, articular e ligamentoso da laringe, da morfologia bucal, faríngea, nasal, além da musculatura da língua, palato, lábios, face e a integridade da articulação temporomandibular ${ }^{(17)}$. Sendo a respiração oral uma adaptação patológica que causa prejuízos significativos ao sistema sensório-motor-oral (lábios, língua, mandíbula, bochecha) e às funções orais do sistema estomatognático, que levam, também, a um comprometimento da voz, deve se propor uma intervenção global, a fim de possibilitar a adequação da comunicação vocal, bem como a adequação do sistema estomatognático.

\section{COMENTÁRIOS FINAIS}

Durante a revisão de literatura, foi possível observar que, na maioria das publicações encontradas, são considerados os aspectos respiração oral e disfonia de maneira isolada. Porém, a respiração oral promove uma série de alterações estruturais significativas que refletem nas funções estomatognáticas, interferindo também na fonação.

Poucos autores relatam a possível correlação entre esses dois parâmetros (respiração oral e disfonia). Observamos, também, que o tema é escasso e que existem poucos artigos que mostram a correlação sistemática entre respiração oral e disfonia.

Foi possível observar que os distúrbios vocais decorrentes da respiração oral são devidos à obstrução nasal, alterações posturais e tônus muscular, tensão músculo-esquelética e, também, especificadamente, tensão cervical.

Portanto, Motricidade Orofacial e Voz apresentam íntima relação, seja por aspectos anatômicos, funcionais, fisiológicos e neuromusculares.

\begin{abstract}
It is known that mouth breathing can lead to several bone, postural, muscular and functional alterations, and also changes on vocal quality and/or vocal behavior. These alterations can compromise oral communication, in which the voice cannot perform its basic role on the transmission of an individual's verbal and emotional message. Individuals with mouth breathing can present hiper or hiponasal resonance, changes on voicing features and hoarseness. From researches on LILACS and SciELO databases, books and specialized magazines about the subject, it was carried a literature review about the relationship between mouth breathing and dysphonia, analyzing the influence of alterations caused by mouth breathing on vocal quality and/or vocal behavior. It was possible observe that mouth breathing promotes several significant structural alterations, which reflect on stomatognathic functions and on phonation. However, few authors report the possible correlation between mouth breathing and dysphonia.
\end{abstract}

Keywords: Voice; Mouth breathing; Dysphonia; Voice quality

\title{
REFERÊNCIAS
}

1. Rehder MI. Inter-relações entre voz e motricidade oral. In: Ferreira LP, Befi-Lopes DM, Limongi SCO, organizadores. Tratado de fonoaudiologia. São Paulo: Roca; 2004. p.59-64.

2. Felício MC. Desenvolvimento normal das funções estomatognáticas. In: Ferreira LP, Befi-Lopes DM, Limongi SCO. Tratado de fonoaudiologia. São Paulo: Roca; 2004. p.193-211.

3. Marchesan IQ. Fundamentos em fonoaudiologia: aspectos clínicos da motricidade oral. Rio de Janeiro: Guanabara Koogan; 1998. Capítulo 4,
Avaliação terapia dos problemas da respiração; p. 23-36.

4. Abreu ACB, Morales DA, Ballo MBJF. A respiração oral influencia o rendimento escolar? Rev CEFAC. 2003;5(1):69-73.

5. Paes C, Vieira, J, Leonel T, Cunha DA. O impacto da respiração oral no comportamento vocal. J Bras Fonoaudiol. 2005;5(23):417-23.

6. Tessitore A. Alterações oromiofuncionais em respiradores orais. In: Ferreira LP, Befi-Lopes DM, Limongi SCO. Tratado de fonoaudiologia. São Paulo: Roca, 2004. p. 261-76. 
7. Motonaga SM, Berte LC, Anselmo-Lima WT. Respiração bucal: causas e alterações no sistema estomatognático. Rev Bras Otorrinolaringol. 2000;66(4);373-9.

8. Di Francesco RC, Passerotii G, Paulucci B, Miniti A. Respiração oral na criança: repercussões diferentes de acordo com o diagnóstico. Rev Bras Otorrinolaringol. 2004;70(5):665-70.

9. Pinho SMR. Fundamentos em fonoaudiologia: tratando os distúrbios da voz. Rio de Janeiro: Guanabara Koogan; 1998. Parte I: Avaliação e tratamento de voz; p. 3-37.

10. Boone DR, McFarlane SC. Distúrbios da voz. Porto Alegre: Artes Médicas, 1994. Capítulo 3, A voz e a terapia vocal; p. 61-98.

11. Kyrillos LCR. Distúrbios da voz em crianças: fatores causais e prevenção. Mundo Saúde. 1995;19(5):177-81.

12. Behlau M, Pontes P. Avaliação e tratamento das disfonias. São Paulo: Lovise, 1995.

13. Hersan RCGP. Avaliação de voz em crianças. Pró-Fono. 1991;3(1):39.

14. Melo ECM, Mattioli FM, Brasil OCO, Behlau M, Pitaluga ACA, Melo DM. Disfonia infantil: aspectos epidemiológicos. Rev Bras Otorrinolaringol 2001;67(6):804-7.

15. Di Francesco RC. Respirador bucal: a visão do otorrinolaringologista. J Bras Fonoaudiol. 1999;1(1):56-60.

16. Pereira FC, Motonaga SM, Faria PM, Matsumoto MAN, Trawitzki LYV, Lima SA, Lima WTA. Avaliação cefalométrica e miofuncional em respiradores bucais. Rev Bras Otorrinolaringol. 2001;67(1):43-9.

17. Meirelles RC. Obstrução nasal e nódulos vocais. Rev Bras Otorrinolaringol. 2001;67(3):387-92.

18. Principato JJ. Upper airway obstruction and craniofacial morphology. Otolaryngol Head Neck Surg. 1991;104(6):881-90.

19. Ferraz MJPC, Nouer DF, Teixeira JR, Bérzin F. Avaliação cefalométrica da posição do osso hióide em crianças respiradoras bucais. Rev Bras Otorrinolaringol. 2007;73(1):47-52.

20. Junqueira PAS, Di Francesco RC, Trezza P, Zeratti FE, Frizzarini R, Faria MEJ. Alterações funcionais do sistema estomatognático pré e pós adenoamigdalectomia. Pró-Fono. 2002;14(1):17-22.

21. Cintra CFSC, Castro FFM, Cintra PPVC. As alterações oro-faciais apresentadas em pacientes respiradores bucais. Rev Bras Alergia Imunopatol. 2000;23(2):78-83.

22. Chami FAI. Avaliação nasofibroscópica e radiológica de pacientes com hiperplasia da amígdala faríngea. Rev Bras Med Otorrinolaringol.1998;5(4):118-24.

23. Martins RHG, Trindade SHK. A criança disfônica: diagnóstico, tratamento e evolução clínica. Rev Bras Otorrinolaringol. 2003;69(6):801-6.

24. Bonatto MTRL, Andrada e Silva MA, Costa HO. A relação entre a respiração e sistema sensório-motor oral em crianças disfônicas. Rev CEFAC. 2004;6(1):58-66.

25. Marchesan IQ, Krakauer LH. A importância do trabalho respiratório na terapia miofuncional. Vol. 2. In: Marchesan IQ, Gomes ICD, Zorzi JL, organizadores. Tópicos em fonoaudiologia. São Paulo: Lovise; 1995. p. 155-160.
26. Nemetz MA, Pontes PAL, Vieira VP, Yazaki RK. Configuração das pregas vestibulares à fonação em adultos com e sem disfonia. Rev Bras Otorrinolaringol. 2005;71(1):6-12.

27. Kooijman PG, De Jong FI, Oudes MJ, Huinck W, Van Acht H, Graamans $\mathrm{K}$. Muscular tension and body posture in relation to voice handicap and voice quality in teachers with persistent voice complaints. Folia Phoniatr Logop. 2005;57(3):134-47.

28. Câmara LBV, Assencio-Ferreira VJ. Efeitos da constrição ânteroposterior na voz. Rev CEFAC. 2000;2(2):97-101.

29. Sataloff TS. Anatomia funcional e fisiologia da voz. In: Sataloff TS, Gould WJ, Spiegel JR. Manual prático de oncocirurgia. Rio de Janeiro: Revinter, 2002. p.63-176.

30. Freitas MR, Pela S, Gonçalves MLR, Fujita RR, Pontes PA, Weckx LLM. Disfonia crônica na infância e adolescência: estudo retrospectivo. Rev Bras Otorrinolaringol. 2000;66(5):480-4.

31. Pinho SMR. Fisiologia da fonação. In: Ferreira LP, Befi-Lopes DM, Limongi SCO. Tratado de fonoaudiologia. São Paulo: Roca, 2004. p.310.

32. Oliveira IB. Avaliação fonoaudiológica da voz: reflexões sobre condutas, com efoques à voz profissional. In: Ferreira LP, Befi-Lopes DM, Limongi SCO. Tratado de fonoaudiologia. São Paulo: Roca, 2004. p. 11-24.

33. Frasson JMD, Magnani MBBA, Nouer DF, Siqueira VCV, Lunardi N. Estudo cefalométrico comparativo entre respiradores nasais e predominantemente bucais. Rev Bras Otorrinolaringol. 2006;72(1):7282.

34. Garcia RAS, Campiotto AR. Distúrbios vocais x distúrbios musculares orais: possíveis relações. Pró-Fono. 1995;7(2):33-9.

35. Corsi SL. Incidência de sinais e sintomas vocais em pacientes com rinite alérgica. Vol. 2. In: Behlau MS, organizador. A voz do especialista. Rio de Janeiro: Revinter; 2001. p. 86-100.

36. Peter GS, Pinho SMR, Assencio-Ferreira VJ. Musculatura extrínseca da laringe e sua participação na produção vocal. Rev CEFAC. 2001;3(2):165-73.

37. Lessa FCR, Enoki C, Feres MFN, Valera FCP, Lima WTA, Matsumoto MAN. Influência do padrão respiratório na morfologia craniofacial. Rev Bras Otorrinolaringol. 2005;71(2):156-60.

38. Ferreira ML. A incidência de respirações bucais em indivíduos com oclusão classe II. J Brás Ortodon Ortop Facial. 1999;4(21):223-40.

39. Oliveira VL, Pinho SM. A qualidade da voz e o trato vocal nos indivíduos de face curta e face longa. In: Pinho SMR. Tópicos em voz. Rio de Janeiro: Guanabara Koogan; 2001. p. 81-8.

40. Gonçalves MI, Weckx LL, Behlau MS, Lederman HM, Lourenço MT, Hirata $\mathrm{CH}$. Exame otorrinolaringológico, cinefluroscopia e análise perceptual auditiva em crianças com indicação de adenoidectomia. Acta Awho. 1989;8:17-20. 